\title{
The Computational Significance of Hausdorff's Maximal Chain Principle
}

\author{
Peter Schuster ${ }^{(\otimes)}(\mathbb{D})$ and Daniel Wessel ${ }^{(\otimes)}(\mathbb{D}$ \\ Dipartimento di Informatica, Università degli Studi di Verona, \\ Strada le Grazie 15, 37134 Verona, Italy \\ \{peter.schuster, daniel.wessel\} @univr.it
}

\begin{abstract}
As a fairly frequent form of the Axiom of Choice about relatively simple structures (posets), Hausdorff's Maximal Chain Principle appears to be little amenable to computational interpretation. This received view, however, requires revision. When attempting to convert Hausdorff's principle into a conservation theorem, we have indeed found out that maximal chains are more reminiscent of maximal ideals than it might seem at first glance. The latter live in richer algebraic structures (rings), and thus are readier to be put under computational scrutiny. Exploiting the newly discovered analogy between maximal chains and ideals, we can carry over the concept of Jacobson radical from a ring to an arbitrary set with an irreflexive symmetric relation. This achievement enables us to present a generalisation of Hausdorff's principle first as a semantic and then as a syntactical conservation theorem. We obtain the latter, which is nothing but the desired computational core of Hausdorff's principle, by passing from maximal chains to paths of finite binary trees of an adequate inductively generated class. In addition to Hausdorff's principle, applications include the Maximal Clique Principle for undirected graphs. Throughout the paper we work within constructive set theory.
\end{abstract}

Keywords: Axiom of Choice - Maximal chain - Maximal ideal · Maximal clique - Jacobson radical $\cdot$ Proof-theoretic conservation · Computational content $\cdot$ Constructive set theory $\cdot$ Finite binary tree Inductive generation

\section{Introduction}

Hausdorff's maximal chain principle asserts that every totally ordered subset of a partially ordered set $S$ is contained in a maximal one. Equivalently, this can be put as a completeness criterion in first-order terms: a chain $C$ is maximal precisely when, for every $x \in S$, if $C \cup\{a\}$ is a chain, then $a \in C$. So a chain $C$ is maximal if and only if, for every $a \in S$, either $a \in C$ or $a$ is incomparable with at least one $b \in C$, i.e.,

$$
a \in C \vee(\exists b \in C)(a \nless b \wedge b \nless a) .
$$

(C) Springer Nature Switzerland AG 2020

M. Anselmo et al. (Eds.): CiE 2020, LNCS 12098, pp. 239-250, 2020.

https://doi.org/10.1007/978-3-030-51466-2_21 
This is somewhat reminiscent of the characterisation of maximal ideals in commutative ring theory [21]. In this setting an ideal $J$ of a commutative unital ring takes the place of $C$, and the respective right-hand disjunct of (1) expresses that the ring element $a$ is invertible modulo $J$. Moreover, it is possible to describe the common part of all maximal ideals in first-order terms. This encodes Krull's Maximal Ideal Theorem as an intersection principle, and yields a notion of Jacobson radical suitable for constructive algebra $[21,31,38]$.

By analogy, we can define the Jacobson radical $\operatorname{Jac}(C)$ of a chain $C$, and prove (assuming the Axiom of Choice $\mathrm{AC}$ ) that $\operatorname{Jac}(C)$ coincides with the intersection of all maximal chains containing $C$. Hence Hausdorff's principle too can be recast as an intersection principle. All this will even be done in a slightly more general fashion. The main point to be stressed is that a simple constructive interpretation is possible, whence the purpose of this paper is twofold: we communicate a new choice principle, and describe its constructive underpinning.

We proceed as follows. In Sect.2, alongside the analogy with ring theory, we describe our concepts of coalition and Jacobson radical. In Sect. 3 we briefly relate this to past work [25-27] on the interplay of single- and multi-conclusion entailment relations $[9,35]$. In Sect. 4 we give a constructive account of complete coalitions by means of a suitable inductively generated class of binary trees. In Sect. 5 we briefly discuss two applications: maximal chains of partially ordered sets, and maximal cliques of undirected graphs. The main results are Proposition 1 and its constructive companion Proposition 3.

\section{Foundations}

The content of this paper is elementary and can be formalised in a suitable fragment of constructive set theory CZF $[2,3]$. Due to the choice of this setting, sometimes certain assumptions have to be made explicit which otherwise would be trivial in classical set theory. For instance, a subset $T$ of a set $S$ is detachable if, for every $a \in S$, either $a \in T$ or $a \notin T$. A set $S$ is finitely enumerable if there is $n \geqslant 0$ and a surjective function $f:\{1, \ldots, n\} \rightarrow S$. We write $\operatorname{Fin}(S)$ for the set of finitely enumerable subsets of $S$. To pin down a rather general, classical intersection principle, and to point out certain of its incarnations, requires some classical logic and the Axiom of Choice (AC) in its classically equivalent form of Zorn's Lemma (ZL) [40]. For simplicity we switch in such a case to classical set theory ZFC, signalling this appropriately.

\section{Coalitions}

Throughout, let $S$ be a set, and let $R$ be an irreflexive symmetric relation on $S$. We say that a subset $C$ of $S$ be a coalition ${ }^{1}$ (with respect to $R$ ) if $\neg a R b$ for all $a, b \in C$. This is the same as demanding that $C$ be $\bar{R}$-connected, which is to say

\footnotetext{
${ }^{1}$ Incidentally, the term "coalition", which we use here for sake of intuition, is standard terminology in game theory to denote a group of agents [39].
} 
that $a \in C$ only if $a \bar{R} b$ for every $b \in C$, where $\bar{R}$ denotes the complementary relation. For instance, the empty subset is a coalition, as is every singleton subset of $S$, by the irreflexivity of $R$. Notice that coalitions are closed under directed union. A coalition $C$ is called complete if, for every $a \in S$,

$$
a \in C \vee(\exists b \in C) a R b .
$$

It is perhaps instructive to read $a R b$ as " $a$ opposes $b$ " (and vice versa, to account for symmetry), under which reading it makes sense to require irreflexivity. A coalition is then a subset of $S$ in which no two members oppose one another. A complete coalition $C$ is such that, given any $a \in S$, this $a$ either belongs to $C$, or else $C$ exhibits a witness $b$ which opposes $a$.

Lemma 1. Every complete coalition is detachable and maximal (with respect to set inclusion) among coalitions. Conversely, with classical logic every maximal coalition is complete.

Proof. Let $C$ be a complete coalition. Since $a \bar{R} b$ for all $a, b \in C$, the second alternative of completeness (2) entails that $a \notin C$; whence $C$ is detachable. As regards $C$ being maximal, let $D$ be a coalition such that $C \subseteq D$ and let $a \in D$. By completeness, either $a \in C$ right away, or else there is $b \in C$ such that $a R b$, but the latter case is impossible as $D$ is a coalition. As regards the converse, if $C$ is a complete coalition and $a \notin C$, then $C^{\prime}=C \cup\{a\}$ cannot, due to maximality of $C$, in turn be a coalition. With classical logic, the latter statement is witnessed by a certain element $b \in C$. This yields completeness.

If $C$ is a coalition, let us write

\section{Comp $/ C$}

for the collection of all complete coalitions that contain $C$, with the special case Comp $=$ Comp $/ \emptyset$. Since every complete coalition is detachable (Lemma 1), these collections are sets due to the presence in $\mathbf{C Z F}$ of the Exponentiation Axiom $[2,3]$.

All this is fairly reminiscent of the characteristics of maximal ideals in ring theory [21]. Given a commutative ring $A$ with 1 , recall from $[12,21]$ that the Jacobson radical [20] of an ideal $J$ of $A$ can be defined as

$$
\operatorname{Jac}(J)=\{a \in A \mid(\forall b \in A)(1 \in\langle a, b\rangle \rightarrow(\exists c \in J) 1 \in\langle b, c\rangle)\},
$$

where sharp brackets denote generated ideals. By plain analogy with the ringtheoretic setting, let us then define the Jacobson radical of an arbitrary subset $C$ of $S$, of course with respect to our default, irreflexive symmetric relation $R$ :

$$
\operatorname{Jac}(C)=\{a \in S \mid(\forall b \in S)(a R b \rightarrow(\exists c \in C) b R c)\} .
$$

In particular, the Jacobson radical of the empty coalition is

$$
\operatorname{Jac}(\emptyset)=\{a \in S \mid(\forall b \in S) a \bar{R} b\} .
$$


Thus, we substitute the property of mutual opposition in (4) for the one of comaximality in (3), i.e., for the property of two ring elements to generate the unit ideal. Assuming AC, the Jacobson radical of an ideal $J$ is the intersection of all maximal ideals that contain $J$ [21]. Similarly, and still with AC, the Jacobson radical of a coalition $C$ turns out to be the intersection of all complete coalitions containing $C$ (Proposition 1).

Lemma 2. The Jacobson radical defines a closure operator on $S$ which restricts to a mapping on coalitions, i.e., if $C$ is a coalition, then so is $\operatorname{Jac}(C)$.

Proof. As for the first statement we only show idempotency, i.e., $\operatorname{Jac}(\operatorname{Jac}(C)) \subseteq$ $\operatorname{Jac}(C)$, where $C \subseteq S$. In fact, if $a \in \operatorname{Jac}(\operatorname{Jac}(C))$ and $b \in S$ is such that $a R b$, then there is $c \in \operatorname{Jac}(C)$ with $c R b$. It follows that there is $c^{\prime} \in C$ such that $b R c^{\prime}$, and so $a \in \operatorname{Jac}(C)$.

As regards the second statement, suppose that $C \subseteq S$ is a coalition, and let $a_{0}, a_{1} \in \operatorname{Jac}(C)$. Assuming that $a_{1} R a_{0}$, since $a_{1} \in \operatorname{Jac}(C)$, there is $c_{0} \in C$ such that $a_{0} R c_{0}$. Since $a_{0} \in \operatorname{Jac}(C)$ too, there is $c_{1} \in C$ such that $c_{0} R c_{1}$, which is in conflict with $C$ being a coalition.

Proposition 1 (ZFC). If $C$ is a coalition, then

$$
\operatorname{Jac}(C)=\bigcap \operatorname{Comp} / C
$$

Proof. Let $a \in \operatorname{Jac}(C)$ and suppose that $D$ is a complete coalition which contains $C$. By completeness, either $a \in D$ right away, or else there is $b \in D$ such that $a R b$. But since $a \in \operatorname{Jac}(C)$, the latter case would imply that there were $c \in C \subseteq D$ with $b R c$, by way of which $D$ would fail to be a coalition after all.

For the right-to-left inclusion we concentrate on the contrapositive. Thus, suppose that $a \notin \operatorname{Jac}(C)$. Accordingly, there is $b$ such that $a R b$ and $C^{\prime}:=C \cup\{b\}$ is a coalition. ZL yields a coalition $D$ which is maximal among those containing $C^{\prime}$. This $D$ is complete by way of being maximal, and it must avoid $a$, because if $a \in D$, then $D$ were not a coalition since $b \in C^{\prime} \subseteq D$ and $a R b$.

Remark 1. The argument in the right-to-left part of the proof of Proposition 1 can also be used in a more affirmative manner. ZL, which is said to be constructively neutral [4], ${ }^{2}$ directly implies that

$$
\bigcap \operatorname{Max} / C \subseteq\{a \in S \mid(\forall b \in S)(a R b \rightarrow \neg(\forall c \in C) b \bar{R} c)\}
$$

where Max $/ C$ denotes the collection of all maximal coalitions over $C$. The crucial direction of Proposition 1 can also be proved in a more direct manner by using Open Induction $[6,11,23]$ in place of Zorn's Lemma. For similar cases see [10, $24,29,30]$.

\footnotetext{
${ }^{2}$ Forms of ZL have been considered over classical [14], intuitionistic [5] as well as constructive set theory $[1,32]$.
} 
By a radical coalition $C$ we understand one which is closed with respect to Jac, i.e., which is such that $\operatorname{Jac}(C)=C$. Clearly, every complete coalition is radical, and by Lemma 2 so is the intersection of an inhabited family of complete coalitions. By Proposition 1, in ZFC the radical coalitions are precisely the intersections of complete coalitions; so in particular

$$
\{a \in S \mid(\forall b \in S) a \bar{R} b\}=\operatorname{Jac}(\emptyset)=\bigcap \text { Comp. }
$$

With Proposition 3 we will give a constructive version of Proposition 1 in Sect. 4, to which end Proposition 2 below will be crucial.

In the following, we write

$$
R(x)=\{y \in S \mid x R y\}
$$

for the image of $x$ under $R$, and use $\operatorname{Jac}(C, x)$ as a shorthand for $\operatorname{Jac}(C \cup\{x\})$.

Proposition 2. The following is provable for the Jacobson radical:

$$
\frac{a \in \operatorname{Jac}(C, x) \quad(\forall y \in R(x)) a \in \operatorname{Jac}(C, y)}{a \in \operatorname{Jac}(C)}
$$

where $a, x \in S$ and $C$ is an arbitrary subset of $S$.

Proof. Given the displayed premises, to check that $a \in \operatorname{Jac}(C)$, consider $b \in S$ such that $a R b$. We need to find $c \in C$ such that $b R c$. The left-hand premise yields $c^{\prime} \in C \cup\{x\}$ such that $b R c^{\prime}$. If $c^{\prime} \in C$, then $c=c^{\prime}$ is as required. In case of $c^{\prime}=x$, the right-hand premise for $y=b$ yields $a \in \operatorname{Jac}(C, b)$. Again with $a R b$ it follows that there is $c \in C \cup\{b\}$ such that $b R c$, whence in fact $c \in C$ since $R$ is irreflexive.

Remark 2. Given a binary relation $R$ on $S$, an $R$-clique is a subset $C$ such that, for every $a \in S$,

$$
a \in C \Leftrightarrow(\forall b \in C) a R b .
$$

Bell's Clique Property asserts that, for any reflexive symmetric relation $R$ on $S$, an $R$-clique exists. This is in fact an intuitionistic equivalent of ZL [5]. Classically, given an irreflexive symmetric relation $R$, every $\bar{R}$-clique is a complete $R$-coalition. Conversely, and constructively, every complete $R$-coalition is an $\bar{R}$ clique. More precisely, a subset $C$ of $S$ is an $\bar{R}$-clique if and only if it is $\bar{R}$ connected as well as $\bar{R}$-saturated, the latter of which is to say that $a \in C$ already if $a \bar{R} b$ for all $b \in C$.

\section{Entailment for Completeness}

Consider on $S$ the relation $\triangleright \subseteq \operatorname{Fin}(S) \times S$ which is defined by the Jacobson radical, i.e., stipulate

$$
U \triangleright a \equiv a \in \operatorname{Jac}(U)
$$


Lemma 2 tells us that this $\triangleright$ is a single-conclusion entailment relation, which is to say that it is reflexive, monotone, and transitive in the following sense:

$$
\frac{U \ni a}{U \triangleright a}(\mathrm{R}) \quad \frac{U \triangleright a}{U, V \triangleright a}(\mathrm{M}) \quad \frac{U \triangleright b \quad U, b \triangleright a}{U \triangleright a}(\mathrm{~T})
$$

where the usual shorthand notation is at work with $U, V \equiv U \cup V$ and $U, b \equiv$ $U \cup\{b\}$. In ZFC, the consequences with respect to $\triangleright$ of a coalition $U \in \operatorname{Fin}(S)$ are semantically determined by the complete coalitions over $U$, i.e.,

$$
(\forall C \in \operatorname{Comp})(C \supseteq U \Longrightarrow a \in C) \Longrightarrow U \triangleright a .
$$

Proposition 2 implies that the following is provable:

$$
\frac{U, x \triangleright a \quad(\forall y \in R(x)) U, y \triangleright a}{U \triangleright a}
$$

This is to say that the infinitary axiom of completeness (2), which in the present context can be put in the form

$$
\vdash x, R(x)
$$

is in fact conservative $[25,26]$ over $\triangleright$. To make this precise requires extending the results of $[25,26]$ to an infinitary setting [36], but upon which those results go through verbatim. We do not require such a development here; an elementary constructive interpretation of Proposition 1 will be given in the following section using instead a suitable inductively generated collection of finite binary trees. For related uses of conservativity see also, e.g., [16,27,28].

\section{Binary Trees for Complete Coalitions}

In this section we carry over the approach recently followed in [34] for prime ideals of commutative rings, so as to accommodate complete coalitions. Readers familiar with dynamical algebra $[13,21,38]$ will draw a connection between the tree methods of [13] and the one employed here.

Let again $S$ be a set. For every $a \in S$ we first introduce a corresponding letter $X_{a}$. Let

$$
\mathcal{S}=\left(S \cup\left\{X_{a} \mid a \in S\right\}\right)^{*}
$$

be the set of finite sequences of elements of $S$ and such letters, with the usual provisos on notation, concatenation, etc. Next, we generate inductively a class $\mathcal{T}$ of finite rooted binary trees $T \subseteq \mathcal{S}$ as follows:

$$
\overline{\{[]\} \in \mathcal{T}} \text { (root) } \quad \frac{T \in \mathcal{T} \quad u \in \operatorname{Leaf}(T) \quad a \in S}{T \cup\left\{u a, u X_{a}\right\} \in \mathcal{T}} \text { (branch) }
$$

As usual, by a leaf we understand a sequence $u \in T$ without immediate successor in $T$. The second rule is to say that, given $T \in \mathcal{T}$, if $u$ is a leaf of $T$, 
then each element $a$ of $S$ gives rise to a new member of $\mathcal{T}$ by way of an additional branching at $u$. More precisely, $u$ gives birth to two children $u a$ and $u X_{a}$. Here is a possible instance, where $a, b \in S$ :

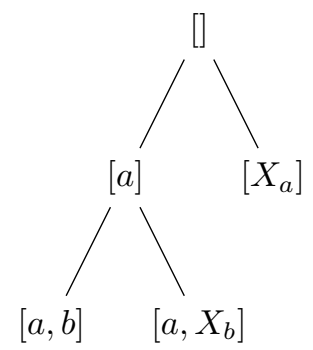

As an auxiliary tool, we further need a sorting function sort $: \mathcal{S} \rightarrow \mathcal{S}$ which gathers all occurring letters $X_{a}$ at the tail of a finite sequence. As the resulting order of the entries won't matter later on, this function may be defined recursively in the simplest manner, as follows:

$$
\begin{aligned}
\operatorname{sort}([]) & =[] \\
\operatorname{sort}(u a) & =a \operatorname{sort}(u) \\
\operatorname{sort}\left(u X_{a}\right) & =\operatorname{sort}(u) X_{a}
\end{aligned}
$$

Last but not least, given a subset $C$ of $S$, we introduce a relation $\vdash_{C}$ between elements of $S$ and sorted finite sequences in $\mathcal{S}$ by defining

$$
\begin{aligned}
& c \Vdash_{C}\left[a_{1}, \ldots, a_{k}, X_{b_{1}}, \ldots, X_{b_{\ell}}\right] \equiv \\
& \left(\forall x_{1}, \ldots, x_{\ell} \in S\right)\left(\bigwedge_{j=1}^{\ell} x_{j} R b_{j} \rightarrow c \in \operatorname{Jac}\left(C, a_{1}, \ldots, a_{k}, x_{1}, \ldots, x_{\ell}\right)\right),
\end{aligned}
$$

where we drop the quantifier in case of $\ell=0$. In particular,

$$
c \Vdash_{C}[] \Leftrightarrow c \in \operatorname{Jac}(C) .
$$

Keeping in mind Proposition 1, with AC the semantics of this relation is that if $u=\left[a_{1}, \ldots, a_{k}, X_{b_{1}}, \ldots, X_{b_{\ell}}\right]$ as above, then $c \Vdash_{C} u$ precisely when, for every simultaneous instantiation of respective opponents $x_{1}, \ldots, x_{\ell}$ of $b_{1}, \ldots, b_{\ell}$, this $c$ is a member of every complete coalition over $C$ that further contains $a_{1}, \ldots, a_{k}, x_{1}, \ldots, x_{\ell}$. The case in which this holds with respect to every leaf of a certain tree $T \in \mathcal{T}$ will later be of particular interest.

With the relation $\Vdash_{C}$ in place, we can now rephrase Proposition 2 as follows.

Lemma 3. Let $a, c \in S$ and let $u \in \mathcal{S}$ be sorted. If $c \Vdash_{C}$ au and $c \Vdash_{C} u X_{a}$, then $c \Vdash_{C} u$.

Proof. Consider $u=\left[a_{1}, \ldots, a_{k}, X_{b_{1}}, \ldots, X_{b_{\ell}}\right]$ and suppose that (i) $c \Vdash_{C}$ au and (ii) $c \Vdash_{C} u X_{a}$. To show that $c \Vdash_{C} u$, let $x_{1} \in R\left(b_{1}\right), \ldots, x_{\ell} \in R\left(b_{\ell}\right)$. We write $C^{\prime}=C \cup\left\{a_{1}, \ldots, a_{k}, x_{1}, \ldots, x_{\ell}\right\}$ and need to check that $c \in \operatorname{Jac}\left(C^{\prime}\right)$. With $x_{1}, \ldots, x_{\ell}$ fixed, premise (i) yields $c \in \operatorname{Jac}\left(C^{\prime}, a\right)$, while (ii) implies that, for every $x \in R(a), c \in \operatorname{Jac}\left(C^{\prime}, x\right)$. Now Proposition 2 implies $a \in \operatorname{Jac}\left(C^{\prime}\right)$. 
Given a subset $C$ and an element $c$ of $S$, let us say that a tree $T \in \mathcal{T}$ terminates for $C$ in $c$ if $c \Vdash_{C}$ sort $(u)$ for every leaf $u$ of $T$. Intuitively, this is to say that, along every path of $T$, no matter how we instantiate indeterminates $X_{b}$ that we might encounter with a corresponding opponent $x$ of $b$, if $C^{\prime}$ is a complete coalition over $C$ and contains the elements we will have collected at the leaf, then $c$ is a member of $C^{\prime}$. The idea is now to fold up branchings by inductive application of Lemma 3, to capture termination by way of the Jacobson radical, and thus to resolve indeterminacy in the spirit of [33].

The following is the constructive counterpart of Proposition 1 and does not require that $C$ be a coalition to start with.

Proposition 3. Let $C$ be a subset and $c$ an element of $S$. The following are equivalent.

1. $c \in \operatorname{Jac}(C)$.

2. There is $T \in \mathcal{T}$ which terminates for $C$ in $c$.

Proof. If $c \in \operatorname{Jac}(C)$, then $c \Vdash_{C}$ [] by (6), which is to say that [] terminates for $C$ in $c$. Conversely, suppose that $T \in \mathcal{T}$ is such that $c \Vdash_{C} \operatorname{sort}(u)$ for every leaf $u$ of $T$. We argue by induction on $T$ to show that $c \in \operatorname{Jac}(C)$. The case $T=[]$ is trivial (6). Suppose that $T$ is the result of a branching at a certain leaf $u$ of an immediate subtree $T^{\prime}$, and suppose further that $c \Vdash_{C} \operatorname{sort}(u a)=a \operatorname{sort}(u)$ as well as $c \Vdash_{C} \operatorname{sort}\left(u X_{a}\right)=\operatorname{sort}(u) X_{a}$ for a certain $a \in S$. Lemma 3 implies that $c \Vdash_{C} \operatorname{sort}(u)$, whence we reduce to $T^{\prime}$, to which the induction hypothesis applies.

Membership in a radical coalition $C$ is thus tantamount to termination.

Remark 3. Very much in the spirit of dynamical algebra $[13,21,37,38]$, every tree $T \in \mathcal{T}$ represents the course of a dynamic argument as if a given coalition were complete. Note that every complete coalition $C_{m}$ of $S$ gives rise to a path through a given tree $T \in \mathcal{T}$. In fact, at each branching, corresponding to an element $a$ of $S$, by way of completeness this $a$ either belongs to $C_{m}$ or else the latter assigns a value to $X_{a}$ in the sense of exhibiting a witness $b \in C_{m}$ for which $a R b$. The entries in the terminal node of this path, with values assigned appropriately, then belong to $C_{m}$. In particular, if $T$ terminates in $c$ for a certain subset $C \subseteq C_{m}$, then $c \in C_{m}$ because $c \in \operatorname{Jac}(C) \subseteq \operatorname{Jac}\left(C_{m}\right)=C_{m}$ by Proposition 3 and the fact that every complete coalition is radical.

Remark 4. In general it cannot be decided effectively, i.e., without using some excluded middle, whether, given $c \in S$ and $C \subseteq S$, there is a tree $T \in \mathcal{T}$ which terminates for $C$ in $c{ }^{3}$ This is due to the constructive character of Proposition 3 and the following Brouwer-style counterexample. Let $\varphi$ be a bounded formula. ${ }^{4}$ Let $S=\{0,1\}$ and put

$$
R_{\varphi}=\{(0,1) \mid \varphi\} \cup\{(1,0) \mid \varphi\} .
$$

\footnotetext{
${ }^{3}$ One of the anonymous referees has kindly drawn our attention to this.

${ }^{4}$ A set-theoretic formula $\varphi$ is bounded if only set-bounded quantifiers $\forall x \in y$ and $\exists x \in y$ occur in $\varphi$.
} 
By definition, this relation clearly is irreflexive and symmetric. Consider now the corresponding Jacobson radical $\operatorname{Jac}(\emptyset)$. It is easy to see that

$$
0 \in \operatorname{Jac}(\emptyset) \quad \Leftrightarrow \quad \neg \varphi .
$$

Therefore, if $\operatorname{Jac}(\emptyset)$ is detachable, then

$$
\neg \varphi \vee \neg \neg \varphi .
$$

This is to say that the Weak Restricted Law of Excluded Middle (WREM) holds.

\section{Applications}

We will now briefly discuss two instantiations of Proposition 1, concerning maximal chains of partially ordered sets and maximal cliques in undirected graphs. In both cases Proposition 3 provides the corresponding constructive underpinning, which we leave to the reader to spell out in detail. Incidentally, the trick is to start with a relation $R$ of which only the complement $\bar{R}$ is the relation one actually one wants to consider. This clearly fits the concept of coalition we are employing.

\section{Hausdorff's Principle}

Let $(S, \leqslant)$ be a partially ordered set. On $S$ we consider the binary relation $R$ of incomparability, which is

$$
a R b \equiv a \nless b \wedge b \nless a,
$$

and for which $\bar{R}$ means comparability. Classically, a coalition for $R$ is nothing but a chain, i.e., a totally ordered subset of $S$, and the complete coalitions are the maximal chains. As regards the Jacobson radical in this setting, Proposition 1 applied to the empty chain yields that

$$
\{a \in S \mid(\forall b \in S)(a \leqslant b \vee b \leqslant a)\}=\bigcap \text { Comp. }
$$

This is a way to rephrase Hausdorff's maximal chain principle [17]. In fact, if $S$ is not totally ordered by $\leqslant$, as witnessed by a certain element $a$ of $S$ incomparable to some $b \in S$, then by (7) and classical logic there is a maximal chain that avoids $a$. Incidentally, this application helps to calibrate Proposition 1, which over classical set theory ZF thus turns out equivalent to AC through Hausdorff's principle $[18,19,22]$.

\section{Maximal Cliques}

Let $G=(V, E)$ be an undirected graph, $V$ being its set of vertices, $E$ its set of edges, i.e., $E$ is a set of unordered pairs of elements of $V$. On the set of vertices we consider the binary relation $R$ of nonadjacency, which is

$$
a R b \equiv a \neq b \wedge\{a, b\} \notin E .
$$


In this setting, classically, a coalition for $R$ is nothing but a clique $^{5}$ [7], i.e., a subset of $V$ every two distinct elements of which are adjacent, and the complete coalitions are the maximal cliques. Concerning the Jacobson radical, Proposition 1 implies that

$$
\{a \in V \mid(\forall b \in V)(a \neq b \rightarrow\{a, b\} \in E)\}=\bigcap \text { Comp. }
$$

Similar to the preceding application, this yields a solution to the problem of finding a maximal clique with AC. ${ }^{6}$

\section{Conclusion}

Hausdorff's Maximal Chain Principle, a forerunner of Zorn's Lemma [8,40], is presumably one of the most well-known order-theoretic forms of the Axiom of Choice. We have seen that the property of a chain to be maximal can be put as a completeness criterion, reminiscent of the case in commutative ring theory for maximal ideals. By analogy with Krull's Theorem for maximal ideals, employing a suitably adapted form of Jacobson radical, it has become possible to put a new variant of Hausdorff's Principle in terms of a universal statement. This has paved the way to a constructive, purely syntactic rereading by means of an inductively defined class of finite binary trees which encode computations along generic maximal chains. It remains to be seen, however, to what extent in a concrete setting our method allows to bypass invocations of Hausdorff's Principle.

Along similar lines, we have carried over the concept of Jacobson radical from commutative rings to the setting of universal algebra and thus to broaden considerably the range of applications that our approach has opened up so far $[33,34]$. In fact, every single-conclusion entailment relation is accompanied by a Jacobson radical which in turn encodes a corresponding maximality principle. In particular, this encompasses the Jacobson radical for distributive lattices [12], commutative rings [31], as well as for propositional theories [15,16]. We keep for future research to put all this under computational scrutiny, and to compare with ours the related methods employed in dynamical algebra [13].

Acknowledgements. The present study was carried out within the projects "A New Dawn of Intuitionism: Mathematical and Philosophical Advances" (ID 60842) funded by the John Templeton Foundation, and "Reducing complexity in algebra, logic, combinatorics - REDCOM" belonging to the programme "Ricerca Scientifica di Eccellenza 2018" of the Fondazione Cariverona. The authors are members of the Gruppo Nazionale per le Strutture Algebriche, Geometriche e le loro Applicazioni (GNSAGA) within the Italian Istituto Nazionale di Alta Matematica (INdAM) (The opinions expressed in this paper are those of the authors and do not necessarily reflect the views of those foundations.). The anonymous referees' careful readings of the manuscript and several helpful remarks and suggestions are gratefully acknowledged.

\footnotetext{
${ }^{5}$ A caveat on terminology: this notion is a priori different from the one used in Bell's Clique Property (cf. Remark 2) but which carries over to graph theory.

${ }^{6}$ Clique problems, e.g., the problem of finding a maximum clique and that of listing all maximal cliques are prominent in finite graph theory and computational complexity theory [7].
} 


\section{References}

1. Aczel, P.: Zorn's Lemma in CZF (2002). Unpublished note

2. Aczel, P., Rathjen, M.: Notes on constructive set theory. Technical report, Institut Mittag-Leffler (2000). Report No. 40

3. Aczel, P., Rathjen, M.: Constructive set theory (2010). https://www1.maths.leeds. ac.uk/ rathjen/book.pdf, book draft

4. Bell, J.L.: Zorn's lemma and complete Boolean algebras in intuitionistic type theories. J. Symb. Log. 62(4), 1265-1279 (1997)

5. Bell, J.L.: Some new intuitionistic equivalents of Zorn's lemma. Arch. Math. Logic 42(8), 811-814 (2003)

6. Berger, U.: A computational interpretation of open induction. In: Titsworth, F. (ed.) Proceedings of the Ninetenth Annual IEEE Symposium on Logic in Computer Science, pp. 326-334. IEEE Computer Society (2004)

7. Bondy, J., Murty, U.: Graph Theory, Graduate Texts in Mathematics, vol. 244. Springer, London (2008)

8. Campbell, P.J.: The origin of "Zorn's lemma". Historia Math. 5, 77-89 (1978)

9. Cederquist, J., Coquand, T.: Entailment relations and distributive lattices. In: Buss, S.R., Hájek, P., Pudlák, P. (eds.) Logic Colloquium 1998. Proceedings of the Annual European Summer Meeting of the Association for Symbolic Logic, Prague, Czech Republic, 9-15 August 1998. Lect. Notes Logic, vol. 13, pp. 127-139. A. K. Peters, Natick, MA (2000)

10. Ciraulo, F., Rinaldi, D., Schuster, P.: Lindenbaum's Lemma via Open Induction. In: Kahle, R., Strahm, T., Studer, T. (eds.) Advances in Proof Theory. PCSAL, vol. 28, pp. 65-77. Springer, Cham (2016). https://doi.org/10.1007/978-3-319-291987_3

11. Coquand, T.: A note on the open induction principle. Technical report, Göteborg University (1997). www.cse.chalmers.se/ coquand/open.ps

12. Coquand, T., Lombardi, H., Quitté, C.: Dimension de Heitmann des treillis distributifs et des anneaux commutatifs. Publications Mathématiques de Besançon. Algèbre et Théorie des Nombres, pp. 57-100 (2006)

13. Coste, M., Lombardi, H., Roy, M.F.: Dynamical method in algebra: effective Nullstellensätze. Ann. Pure Appl. Logic 111(3), 203-256 (2001)

14. Felgner, U.: Untersuchungen über das Zornsche Lemma. Compos. Math. 18, 170180 (1967)

15. Fellin, G.: The Jacobson radical: from algebra to logic. Master's thesis. Università di Verona, Dipartimento di Informatica (2018)

16. Fellin, G., Schuster, P., Wessel, D.: The Jacobson radical of a propositional theory. In: Piecha, T., Schroeder-Heister, P. (eds.) Proof-Theoretic Semantics: Assessment and Future Perspectives. Proceedings of the Third Tübingen Conference on ProofTheoretic Semantics, 27-30 March 2019, pp. 287-299. University of Tübingen (2019). http://dx.doi.org/10.15496/publikation-35319

17. Hausdorff, F.: Grundzüge der Mengenlehre. Verlag von Veit \& Comp, Leipzig (1914)

18. Herrlich, H.: Axiom of Choice, Lecture Notes in Mathematics, vol. 1876. Springer, Berlin (2006). https://doi.org/10.1007/11601562

19. Howard, P., Rubin, J.: Consequences of the Axiom of Choice. American Mathematical Society, Providence (1998)

20. Jacobson, N.: The radical and semi-simplicity for arbitrary rings. Am. J. Math. 67(2), 300-320 (1945). https://doi.org/10.2307/2371731 
21. Lombardi, H., Quitté, C.: Commutative Algebra: Constructive Methods. Finite Projective Modules, Algebra and Applications, vol. 20. Springer, Netherlands (2015). https://doi.org/10.1007/978-94-017-9944-7

22. Moschovakis, Y.: Notes on Set Teory. Undergraduate Texts in Mathematics, Second edn. Springer, New York (2006)

23. Raoult, J.C.: Proving open properties by induction. Inform. Process. Lett. 29(1), 19-23 (1988)

24. Rinaldi, D., Schuster, P.: A universal Krull-Lindenbaum theorem. J. Pure Appl. Algebra 220, 3207-3232 (2016)

25. Rinaldi, D., Schuster, P., Wessel, D.: Eliminating disjunctions by disjunction elimination. Bull. Symb. Logic 23(2), 181-200 (2017)

26. Rinaldi, D., Schuster, P., Wessel, D.: Eliminating disjunctions by disjunction elimination. Indag. Math. (N.S.) 29(1), 226-259 (2018)

27. Rinaldi, D., Wessel, D.: Cut elimination for entailment relations. Arch. Math. Logic 58(5-6), 605-625 (2019)

28. Schlagbauer, K., Schuster, P., Wessel, D.: Der Satz von Hahn-Banach per Disjunktionselimination. Confluentes Math. 11(1), 79-93 (2019)

29. Schuster, P.: Induction in algebra: a first case study. In: 2012 27th Annual ACM/IEEE Symposium on Logic in Computer Science, LICS 2012. Dubrovnik, Croatia, pp. 581-585. IEEE Computer Society Publications (2012)

30. Schuster, P.: Induction in algebra: a first case study. Log. Methods Comput. Sci. $\mathbf{9}(3), 20$ (2013)

31. Schuster, P., Wessel, D.: Syntax for semantics: Krull's maximal ideal theorem. In: Heinzmann, G., Wolters, G. (eds.) Paul Lorenzen: Mathematician and Logician. Springer, forthcoming

32. Schuster, P., Wessel, D.: A general extension theorem for directed-complete partial orders. Rep. Math. Logic 53, 79-96 (2018)

33. Schuster, P., Wessel, D.: Resolving finite indeterminacy: a definitive constructive universal prime ideal theorem. In: Proceedings of the 35th Annual ACM/IEEE Symposium on Logic in Computer Science (LICS 2020), 8-11 July 2020, Saarbrücken, Germany. ACM, New York (2020)

34. Schuster, P., Wessel, D., Yengui, I.: Dynamic evaluation of integrity and the computational content of Krull's lemma (2019, preprint)

35. Scott, D.: Completeness and axiomatizability in many-valued logic. In: Henkin, L., Addison, J., Chang, C., Craig, W., Scott, D., Vaught, R. (eds.) Proceedings of the Tarski Symposium (Proceedings of Symposia in Pure Mathematics, vol. XXV, University of California, Berkeley, California (1971). pp. 411-435. American Mathematical Society, Providence (1974)

36. Wessel, D.: Points, ideals, and geometric sequents. Technical report, University of Verona (2018)

37. Yengui, I.: Making the use of maximal ideals constructive. Theoret. Comput. Sci. 392, 174-178 (2008)

38. Yengui, I.: Constructive Commutative Algebra. Projective Modules Over Polynomial Rings and Dynamical Gröbner Bases, Lecture Notes in Mathematics, vol. 2138. Springer, Cham (2015). https://doi.org/10.1007/978-3-319-19494-3

39. Young, H.P., Zamir, S. (eds.): Handbook of Game Theory, Volume 4. Handbooks in Economics, 1st edn. North-Holland, Amsterdam (2015)

40. Zorn, M.: A remark on method in transfinite algebra. Bull. Am. Math. Soc. 41, 667-670 (1935) 